\title{
Occurrence and duration of various operational definitions of sedentary bouts and cross-sectional associations with cardiometabolic health indicators: The ENERGY-project
}

\author{
T.M. Altenburg ${ }^{\text {a,* }}$, M. de Niet ${ }^{\mathrm{b}}$, M. Verloigne $^{\mathrm{c}}$, I. De Bourdeaudhuij ${ }^{\mathrm{c}}$, O. Androutsos ${ }^{\mathrm{d}}$, Y. Manios ${ }^{\mathrm{d}}$, E. Kovacs ${ }^{\mathrm{e}}$, \\ B. Bringolf-Isler ${ }^{\text {f,g, }}$, J. Brug ${ }^{\text {h }}$, M.J.M.Chinapaw ${ }^{\mathrm{a}}$ \\ a VU University Medical Center, EMGO Institute for Health and Care Research, Department of Public and Occupational Health, Amsterdam, The Netherlands \\ b Science Support, Utrecht, The Netherlands \\ c Ghent University, Department of Movement and Sport Sciences, Ghent, Belgium \\ d Department of Nutrition-Dietetics, School of Health Science and Education, Harokopio University Athens, Athens, Greece \\ e Department of Paediatrics, University of Pécs, Pécs, Hungary \\ ${ }^{\mathrm{f}}$ Department of Epidemiology and Public Health, Swiss TPH, Basel, Switzerland \\ ${ }^{g}$ University of Basel, Basel, Switzerland \\ ${ }^{\mathrm{h}}$ VU University Medical Center, EMGO Institute for Health and Care Research, Department of Epidemiology E Biostatistics, Amsterdam, The Netherlands
}

\section{A R T I C L E I N F O}

Available online 20 December 2014

\section{Keywords:}

Sedentary bout

Sedentary time

Body composition

Fasting blood

Youth

\begin{abstract}
A B S T R A C T
Objective. This study examined the occurrence and duration of sedentary bouts and explored the crosssectional association with health indicators in children applying various operational definitions of sedentary bouts.

Methods. Accelerometer data of 647 children (10-13 years old) were collected in five European countries. We analyzed sedentary time $(<100 \mathrm{cpm})$ accumulated in bouts of at least $5,10,20$ or 30 min based on four operational definitions, allowing 0,30 or $60 \mathrm{~s} \geq 100 \mathrm{cpm}$ within bouts. Health indicators included anthropometrics (i.e. waist circumference and body mass index (BMI)) and in a subsample from two European countries $(\mathrm{n}=112)$ fasting capillary blood levels of glucose, C-peptide, high-density- and low-density cholesterol, and triglycerides. Data collection took place from March to July 2010. Associations were adjusted for age, gender, moderate-to-vigorous physical activity, total wear time and country.

Results. Occurrence of sedentary bouts varied largely between the various definitions. Children spent most of their sedentary time in bouts of $\geq 5$ min while bouts of $\geq 20$ min were rare. Linear regression analysis revealed few significant associations of sedentary time accumulated in bouts of $\geq 5-30 \mathrm{~min}$ with health indicators. Moreover, we found that more associations became significant when allowing no tolerance time within sedentary
\end{abstract} bouts.

Conclusion. Despite a few significant associations, we found no convincing evidence for an association between sedentary time accumulated in bouts and health indicators in 10-13 year old children.

(c) 2015 The Authors. Published by Elsevier Inc. This is an open access article under the CC BY license (http://creativecommons.org/licenses/by/4.0/).

\section{Introduction}

Recent experimental studies in normal weight young (Altenburg et al., 2013; Peddie et al., 2013) and overweight adults (Dunstan et al., 2012 ) indicate that, compared to uninterrupted sitting, brief, light- or

\footnotetext{
* Corresponding author at: VU University Medical Center, EMGO Institute for Health and Care Research, Department of Public and Occupational Health, van der Boechorststraat 7, 1081 BT, Amsterdam, The Netherlands. Fax: +31204448387.

E-mail addresses: t.altenburg@vumc.nl (T.M. Altenburg), markdeniet@gmail.com (M. de Niet), Maite.Verloigne@Ugent.be (M. Verloigne), Ilse.Debourdeaudhuij@UGent.be (I. De Bourdeaudhuij), oandrou@hua.gr (O. Androutsos), manios@hua.gr (Y. Manios), kovacs.eva.katalin@pte.hu (E. Kovacs), Bettina.Bringolf@unibas.ch (B. Bringolf-Isler), j.brug@vumc.nl (J. Brug), m.chinapaw@vumc.nl (C. M.J.M.).
}

moderate-intensity breaks during prolonged sitting (i.e. 1 min $40 \mathrm{~s}$ breaks every $30 \mathrm{~min}$ ) may attenuate cardiometabolic risk. These studies support the hypothesized mechanism that prolonged sitting leads to the loss of contractile stimulation in weight-bearing muscles, which suppresses skeletal muscle lipoprotein lipase (LPL) activity, leading to a prolonged time in which cellular metabolism substrates are present in the vascular compartments (Bey and Hamilton, 2003; Hamilton et al., 2004). These effects may contribute to the development of cardiovascular diseases. In contrast to studies in adults, brief light-intensity interruptions in sitting did not result in measurable changes in cardiometabolic indicators in 10-14 year old children (Saunders et al., 2013a).

Currently, few epidemiological studies have examined accelerometerbased sedentary bouts and breaks in sedentary time in children (Colley 
et al., 2013; Carson and Janssen, 2011; Carson et al., 2014; Harrington et al., 2011; Saunders et al., 2013b). Although all these studies used similar accelerometer-based data (i.e. three studies used Actigraph models, one study used Actical and one study used ActivPAL), they used different operational definitions of sedentary bouts and breaks in sedentary time (Carson and Janssen, 2011; Harrington et al., 2011; Colley et al., 2013; Carson et al., 2014; Saunders et al., 2013b). In these studies, sedentary bout definitions ranged from periods of $15 \mathrm{~s}$ classified as sitting/ lying (Harrington et al., 2011) to periods of more than 120 min below the frequently used accelerometer cut-point for sedentary activity of $100 \mathrm{cpm}$ (Colley et al., 2013). Strikingly, in two studies, up to a total of $20 \%$ of minutes within a sedentary bout was tolerated above this cutpoint (Carson and Janssen, 2011; Colley et al., 2013). This implies that, for example, if 6 out of 30 min were not spent sedentary, this was still considered a sedentary bout. Importantly, if such interruptions in sedentary time attenuate detrimental effects of prolonged sedentary time (Dunstan et al., 2012; Altenburg et al., 2013; Peddie et al., 2013), we believe that such tolerance minutes should not be accepted in the operational definition of a sedentary bout. Definitions of breaks in sedentary time varied from periods of more than five seconds (Carson et al., 2014) to periods of more than one minute (Saunders et al., 2013b). One study defined a break as each 15-second epoch above $100 \mathrm{cpm}$ within a sedentary bout (Carson and Janssen, 2011).

As a consequence of these different operational definitions of what a sedentary bout is, estimates of sedentary patterns vary largely between studies, making comparison pointless. For example, Harrington et al., (2011) reported that female adolescents (aged 15-18 years) accumulated their weekday sedentary time in on average 53 sedentary bouts of on average 10.3 minutes. Carson et al. (2014) reported that 11 year old children spent on average $240 \mathrm{~min} /$ day in bouts lasting $1-4 \mathrm{~min}$, $91 \mathrm{~min} /$ day in bouts lasting 5-9 $\mathrm{min}, 60 \mathrm{~min} /$ day in bouts lasting 10$19 \mathrm{~min}, 24 \mathrm{~min} /$ day in bouts lasting $20-29 \mathrm{~min}$ and $33 \mathrm{~min} /$ day in bouts lasting $30+$ minutes.

Additionally, the few studies that examined the health effects of sedentary patterns in children and adolescents - varying in age between 6 and 19 years (46-55\% males) - (Carson and Janssen, 2011; Carson et al., 2014; Colley et al., 2013; Saunders et al., 2013b), found inconsistent evidence for an association of sedentary time accumulated in bouts (number and/or duration) in sedentary time with health indicators in children. The different operational definitions of bouts and breaks in sedentary time may be one explanation for these inconsistent findings.

The aim of the present study was therefore to examine the occurrence and duration of sedentary bouts using various operational definitions in 10-13 year old children. Additionally, we explored the association of sedentary time accumulated in bouts according to these operational definitions with cardiometabolic health indicators. Specifically, we examined sedentary bouts of at least $5,10,20$ or $30 \mathrm{~min}$ below $100 \mathrm{cpm}$ in combination with allowing zero and 30 or $60 \mathrm{~s}$ above $100 \mathrm{cpm}$ in a sedentary bout (i.e. tolerance time). Cardiometabolic health indicators included primarily waist circumference and body mass index (BMI), and secondary fasting blood levels of glucose, C-peptide, high-density- and low-density cholesterol, and triglycerides. We hypothesized stronger associations with longer sedentary bout duration and less tolerance within the sedentary bout.

\section{Methods}

\section{Study design and sample}

The study sample consisted of 10-13 year old children and their parents who were enrolled in the ENERGY (EuropeaN Energy balance Research to prevent excessive weight Gain among Youth) cross-sectional survey (van Stralen et al., 2011), as part of the ENERGY-project (Brug et al., 2010). For the current analyses, participants were included from five of the participating countries (i.e. Belgium, Greece, Hungary, Switzerland and the Netherlands) where accelerometer data were obtained. The selection of schools for accelerometer data collection was based on logistic planning of the data collection as well as the willingness of the schools. The data collection took place between March and July 2010, with accelerometer data being collected in approximately 200 children per country. For details see elsewhere (Yildirim et al., 2011). After applying evidence-based data reduction procedures (Chinapaw et al., 2014), we had valid accelerometer data of 647 children. Anthropometrics were collected for all children participating in the accelerometer study from all five countries, but only children from Hungary and the Netherlands who participated in the accelerometer study were asked to provide blood samples. Of the children with valid accelerometer data, fasting blood samples were available for 112 children. The study was approved by the ethics committees in each participating country and is in compliance with the Declaration of Helsinki. All participants and their parents provided written informed consent prior to their inclusion in the study.

\section{Sedentary time and physical activity}

Children were asked to wear an Actigraph accelerometer (models GT1M and Actitrainer) on their waist (right side) during all waking hours, apart from when engaged in water-based activities, for at least six consecutive days. The uniaxial output of these accelerometers is compatible (Robusto and Trost, 2012). A 15-second epoch length was selected to accurately capture the pattern of high intensity and short duration physical activity (Bailey et al., 2013). A detailed description of the accelerometer protocol is described elsewhere (Yildirim et al., 2011).

Raw accelerometer data were analyzed using a customized software program developed in MATLAB. For inclusion in the data analysis, each participant needed a minimum of six days with at least eight valid hours, including at least one weekend day. Periods of more than 60 min of consecutive zero counts were defined as non-wear time and excluded from data analysis (Chinapaw et al., 2014). We selected a cut-point of $<100 \mathrm{cpm}$ to define sedentary time (Trost et al., 2011; Fischer et al., 2012). Total sedentary time (min) was calculated by summing each 15 -second epoch below $100 \mathrm{cpm}$. Moderate-to-vigorous physical activity (MVPA) was defined at a cut-point of $\geq 3000 \mathrm{cpm}$ (Treuth et al., 2004).

Sedentary bouts were defined as periods of consecutive minutes below $100 \mathrm{cpm}$. Using our MATLAB program, we analyzed sedentary time in bouts of at least $5,10,20$ or $30 \mathrm{~min}$ in four separate analyses, allowing 0,30 or 60 (accumulative) tolerance seconds within all different sedentary bout duration (i.e. 12 separate analyses). Time spent in sedentary bouts was calculated as sedentary time ( $\mathrm{min}$ ) accumulated in bouts of at least $5,10,20$ or $30 \mathrm{~min}$. This variable was not averaged into min/day or min/week, as it would have led to a loss of crucial information on bout duration (i.e. sedentary time accumulated in shorter vs. longer bouts). Additionally, the total number of sedentary bouts (per day) of at least 5, 10, 20 or 30 min was calculated.

\section{Indicators of cardiometabolic health}

\section{Anthropometrics}

Data on body height, weight and waist circumference (WC) were collected according to standardized procedures (van Stralen et al., 2011). Participants were measured in light clothing and without shoes. Height $(\mathrm{cm})$ was measured to the nearest $0.1 \mathrm{~cm}$ using a SECA Leicester Portable stadiometer. Body weight $(\mathrm{kg})$ was measured to the nearest $0.1 \mathrm{~kg}$ using a calibrated electronic scale SECA 861. WC $(\mathrm{cm})$ was used as indicator of abdominal fatness and was measured to the nearest $0.1 \mathrm{~cm}$ with the SECA 201 measuring band. Body mass index (BMI) was calculated as weight divided by height squared $\left(\mathrm{m}^{2}\right)$ and was used to assess overweight or obesity, using the Cole criteria (Cole et al., 2000).

\section{Fasting blood samples}

Children in Hungary and the Netherlands were asked to fast the evening prior to the morning of blood collection. Fasting samples were taken between 8:00 and 8:30 A.M. and children were offered breakfast afterwards. Capillary blood samples were collected in a validated collection kit developed for ambulatory purposes (Demecal, The Netherlands (Gootjes et al., 2009)). Following a finger prick, approximately $60 \mu$ of capillary blood was absorbed by a sponge and transferred to a collection tube containing $220 \mu$ dilution buffer. After mixing, the diluted blood was filtered, producing approximately 8 times diluted isolated plasma. Fasting plasma glucose, C-peptide, total cholesterol, lowdensity lipoprotein cholesterol (LDL-C), high-density lipoprotein cholesterol (HDL-C) and triglycerides were determined. 
Statistics

All statistical procedures were performed using SPSS software (version 18.0). A standardized continuously distributed variable ( $z$-score) for clustered cardiometabolic risk was computed from WC, glucose, C-peptide, LDL-C, HDL-C and triglycerides (Altenburg et al., 2012; Chinapaw et al., 2012; Andersen et al., 2006). Each individual cardiometabolic health indicator was converted to $z$-scores and stratified by gender. HDL-C was then multiplied by -1 , since it is inversely related to cardiometabolic risk. For each individual, the average of z-scores of health indicators was calculated to construct the clustered cardiometabolic risk score, with a higher score indicating a higher risk.

Descriptives were presented as mean $\pm S D$ for baseline sample characteristics and median [interquartile range] for sedentary bout descriptives. Multiple linear regression analyses were used to examine the association of total sedentary time and sedentary time accumulated in bouts of different durations with indicators of cardiometabolic health. First, the association of total sedentary time with cardiometabolic indicators was assessed. Second, the association of sedentary time accumulated in sedentary bouts of at least $5,10,20$ or 30 min, respectively, and allowing 0,30 or 60 s tolerance time, respectively, with cardiometabolic indicators was assessed. All associations were adjusted for age, gender, MVPA time, total wear time and country. The associations with fasting blood levels were additionally adjusted for WC (except when clustered cardiometabolic risk was the outcome). Significance level was set at $\mathrm{p}<0.05$.

\section{Results}

Table 1 shows the sample characteristics. Children ( $48 \%$ boys) were on average $11.6( \pm 0.8)$ years old, with $17.3 \%$ being defined as overweight and $4.3 \%$ as obese. Children had on average $6.4( \pm 0.5)$ valid accelerometer days with on average $816( \pm 78)$ minutes valid wear time. They spent on average $511( \pm 84) \mathrm{min} /$ day sedentary and $36( \pm 17) \mathrm{min} /$ day on MVPA (data not shown).

Table 2 shows the descriptives for sedentary bouts according to the different definitions. Logically, sedentary time was higher when allowing sedentary in bouts of shorter duration and tolerating more time above $100 \mathrm{cpm}$ within the bouts. For example, when summing sedentary bouts of $5 \mathrm{~min}$ and allowing $60 \mathrm{~s}$ tolerance time within these bouts, accumulated sedentary time was on average $426 \mathrm{~min}$. When allowing zero tolerance time within these 5-minute bouts, accumulated sedentary time decreased to on average $225 \mathrm{~min}$. When summing bouts of $30 \mathrm{~min}$ and allowing zero tolerance time within these bouts, total accumulated sedentary time was on average only $32 \mathrm{~min}$. Furthermore, children spent most of their time sedentary in bouts of short duration (at least $5 \mathrm{~min}$ ) while sedentary bouts of at least 20 min were rare.

Table 1

Baseline characteristics of 647 European 10-13 year old children (48\% boys) (ENERGY study, March-July 2010).

\begin{tabular}{|c|c|}
\hline & Mean (SD) \\
\hline Age, years & $11.6(0.8)$ \\
\hline $\mathrm{WC}, \mathrm{cm}$ & $65.6(8.6)$ \\
\hline BMI, $\mathrm{kg} / \mathrm{cm}^{2}$ & $18.8(3.3)$ \\
\hline \multicolumn{2}{|l|}{ Weight status, \% } \\
\hline Overweight & 17.3 \\
\hline Obese & 4.3 \\
\hline Glucose $(\mathrm{mmol} / \mathrm{l})^{1}$ & $4.6(0.5)$ \\
\hline Total cholesterol $(\mathrm{mmol} / \mathrm{l})^{1}$ & $3.7(0.7)$ \\
\hline LDL-C $(\mathrm{mmol} / \mathrm{l})^{1}$ & $1.9(0.6)$ \\
\hline $\mathrm{HDL}-\mathrm{C}(\mathrm{mmol} / \mathrm{l})^{1}$ & $1.2(0.3)$ \\
\hline Triglycerides $(\mathrm{mmol} / \mathrm{l})^{1}$ & $0.8(0.5)$ \\
\hline C-peptide $(\mathrm{mmol} / \mathrm{l})^{1,2}$ & $0.6(0.3)$ \\
\hline Cardiometabolic risk score $^{2}$ & $-0.0004(0.6)$ \\
\hline
\end{tabular}

BMI, body mass index; HDL-C, high-density lipoprotein cholesterol; LDL-C,

low-density lipoprotein cholesterol; WC, waist circumference.

1 Subsample of 112 children;

${ }^{2} \mathrm{~N}=111$.

\section{Table 2}

Descriptives (median [interquartile range]) for sedentary time accumulated in bouts of at least 5,10, 20 or $30 \mathrm{~min}$, and including 0,30 or 60 s tolerance time, of 647 European 10-13 year old children (ENERGY study, March-July 2010).

\begin{tabular}{|c|c|c|}
\hline & $\begin{array}{l}\text { Sedentary time accumulated } \\
\text { in bouts (min/day) }\end{array}$ & $\begin{array}{l}\text { Total number of sedentary } \\
\text { bouts per day }\end{array}$ \\
\hline \multicolumn{3}{|c|}{5 min sedentary bout } \\
\hline 60 s tolerance & $426[373 ; 488]$ & $39[35 ; 43]$ \\
\hline 30 s tolerance & $348[294 ; 410]$ & $33[29 ; 37]$ \\
\hline $0 \mathrm{~s}$ tolerance & $225[181 ; 284]$ & $22[18 ; 26]$ \\
\hline \multicolumn{3}{|c|}{10 min sedentary bout } \\
\hline 60 s tolerance & $292[238 ; 360]$ & $17[14 ; 20]$ \\
\hline 30 s tolerance & $221[172 ; 284]$ & $13[10 ; 16]$ \\
\hline $0 \mathrm{~s}$ tolerance & $127[92 ; 173]$ & $7[5 ; 9]$ \\
\hline \multicolumn{3}{|c|}{20 min sedentary bout } \\
\hline 60 s tolerance & $146[105 ; 204]$ & $5[4 ; 7]$ \\
\hline 30 s tolerance & $105[71 ; 149]$ & $3[2 ; 5]$ \\
\hline $0 \mathrm{~s}$ tolerance & $56[36 ; 83]$ & $2[1 ; 3]$ \\
\hline \multicolumn{3}{|c|}{30 min sedentary bout } \\
\hline 60 s tolerance & $80[54 ; 125]$ & $2[1 ; 3]$ \\
\hline 30 s tolerance & $57[35 ; 91]$ & $1[1 ; 2]$ \\
\hline $0 \mathrm{~s}$ tolerance & $32[18 ; 52]$ & $1[0 ; 1]$ \\
\hline
\end{tabular}

Table 3 shows the associations of total sedentary time and Table 4 shows the associations of sedentary time accumulated according to the different definitions with indicators of cardiometabolic risk. Total sedentary time was significantly, positively associated with C-peptide ( $b=0.0003,95 \% \mathrm{CI}=[0.0001 ; 0.0005])$, but not with any of the other indicators of cardiometabolic health (Table 3). Regarding sedentary time accumulated in bouts, of at least $5,10,20$ or $30 \mathrm{~min}$, we found a number of small significant, though rather weak, associations (Table 4). Additionally, we found that the number of significant associations increased when allowing less tolerance seconds within sedentary bouts (i.e. zero vs. 30 or $60 \mathrm{~s}$ tolerance, indicating the most strict sedentary bout definition; Table 4). For example, 60 min more sedentary time accumulated in bouts of at least $5 \mathrm{~min}$, and allowing zero tolerance seconds was associated with a $0.12 \mathrm{~cm}$ higher waist circumference, a $0.012 \mathrm{mmol} / \mathrm{l}$ higher level of C-peptide and a 0.018 higher cardiometabolic risk score. However, when allowing 60 s tolerance within the bouts, 60 min more sedentary time accumulated in bouts of at least $5 \mathrm{~min}$ was only significantly associated with a $0.0012 \mathrm{mmol} / \mathrm{l}$ higher level of C-peptide.

Table 3

Association (beta $(95 \% \mathrm{CI})$ ) of total sedentary time ( $\mathrm{min}$ ) with anthropometrics ( $\mathrm{n}=647$; from 5 European countries) and cardiometabolic risk $(\mathrm{n}=112$; from 2 European countries) in 10-13 year old children (ENERGY study, March-July 2010).

\begin{tabular}{lc}
\hline & Total sedentary time \\
\hline WC & $.000(-.002 ; .002)$ \\
BMI & $.000(-.001 ; .001)$ \\
Glucose & $.0002(-.0001 ; .0004)$ \\
C-peptide & $.0003(.0001 ; .0005)^{*}$ \\
Total cholesterol & $.0000(-.0004 ; .0005)$ \\
LDL-C & $.0002(-.0002 ; .001)$ \\
HDL-C & $.0001(-.0000 ; .0003)$ \\
Triglycerides & $-.0001(-.0004 ; .0002)$ \\
Cardiometabolic risk score ${ }^{1}$ & $.0002(-.0002 ; .001)$ \\
\hline
\end{tabular}

Total sedentary time displayed in total minutes, to make the associations comparable to those of sedentary time accumulated in bouts (i.e. Table 4).

BMI, body mass index; LDL-C, low-density lipoprotein cholesterol; HDL-C, high-density lipoprotein cholesterol; WC, waist circumference.

All associations are adjusted for age, gender, MVPA time, total wear time and country. Except when clustered cardiometabolic risk was the outcome, associations were additionally adjusted for WC.

$1 \mathrm{~N}=111$.

* Indicates statistical significant associations $(\mathrm{p}<0.05)$. 
Table 4

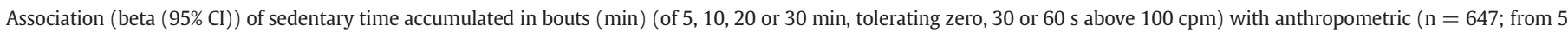
European countries) cardiometabolic risk $(\mathrm{n}=112$; from 2 European countries) in 10-13 year old children (ENERGY study, March-July 2010).

\begin{tabular}{|c|c|c|c|c|}
\hline & $\begin{array}{l}\text { Accumulated sedentary time - } \\
\text { in } \geq 5 \text { min bouts }\end{array}$ & $\begin{array}{l}\text { Accumulated sedentary time - } \\
\text { in } \geq 10 \text { min bouts }\end{array}$ & $\begin{array}{l}\text { Accumulated sedentary time - } \\
\text { in } \geq 20 \text { min bouts }\end{array}$ & $\begin{array}{l}\text { Accumulated sedentary time - } \\
\text { in } \geq 30 \text { min bouts }\end{array}$ \\
\hline \multicolumn{5}{|l|}{60 s tolerance } \\
\hline WC & $.001(-.001 ; .002)$ & $.001(-.000 ; .002)$ & $.001(-.000 ; .002)$ & $.001(-.001 ; .003)$ \\
\hline BMI & $.000(-.001 ; .001)$ & $.000(-.000 ; .001)$ & $.000(-.000 ; .001)$ & $.000(-.000 ; .001)$ \\
\hline Glucose & $.0001(-.0001 ; .0003)$ & $.0002(-.0000 ; .0004)$ & $.0001(-.0001 ; .0004)$ & $-.0001(-.0001 ; .0004)$ \\
\hline C-peptide & $.0002(.0001 ; .0003)^{*}$ & $.0002(.0000 ; .0003)^{*}$ & $.0002(.0000 ; .0003)^{*}$ & $.0001(-.0001 ; .0003)$ \\
\hline Total cholesterol & $.0001(-.0003 ; .0004)$ & $.0001(-.0002 ; .0003)$ & $.0001(-.0002 ; .0004)$ & $.0001(-.0002 ; .0001)$ \\
\hline LDL-C & $.0001(-.0001 ; .0004)$ & $.0001(-.0002 ; .0004)$ & $.0002(-.0001 ; .0004)$ & $.0002(-.0001 ; .0001)$ \\
\hline HDL-C & $.0001(-.0000 ; .0002)$ & $.0001(-.0000 ; .0002)$ & $.0001(-.0001 ; .0002)$ & $.0000(-.0004 ; .0001)$ \\
\hline Triglycerides & $-.0000(-.0002 ; .0002)$ & $-.0000(-.0002 ; .0002)$ & $.0001(-.0003 ; .0002)$ & $-.0002(-.0001 ; .0003)$ \\
\hline Cardiometabolic risk score $^{1}$ & $.0002(-.0001 ; .0005)$ & $.0002(-.0000 ; .0005)$ & $.0003(-.000 ; .001)$ & $.0003(-.0001 ; .001)$ \\
\hline \multicolumn{5}{|l|}{30 s tolerance } \\
\hline WC & $.001(-.000 ; .002)$ & $.001(-.000 ; .003)$ & $.001(-.000 ; .003)$ & $.001(-.001 ; .003)$ \\
\hline BMI & $.000(-.000 ; .001)$ & $.000(-.000 ; .001)$ & $.001(-.000 ; .001)$ & $.001(-.000 ; .001)$ \\
\hline Glucose & $.0001(-.002 ; .002)$ & $.0001(-.0001 ; .0004)$ & $.0002(-.0001 ; .0004)$ & $.0001(-.0002 ; .0004)$ \\
\hline C-peptide & $.0002(.0000 ; .0003)^{*}$ & $.0002(.0000 ; .0003)^{*}$ & $.0002(-.0000 ; .0003)$ & $-.0000(-.0002 ; .0002)$ \\
\hline Total cholesterol & $.0001(-.0002 ; .0004)$ & $.0001(-.0002 ; .0004)$ & $.0002(-.0002 ; .0001)$ & $.0002(-.0003 ; .0001)$ \\
\hline LDL-C & $.0001(-.0001 ; .0004)$ & $.0001(-.0001 ; .0004)$ & $.0003(-.0000 ; .0001)$ & $.0003(-.0001 ; .0001)$ \\
\hline HDL-C & $.0001(-.0001 ; .0002)$ & $.0001(-.0001 ; .0002)$ & $.0001(-.0001 ; .0002)$ & $.0000(-.0002 ; .0000)$ \\
\hline Triglycerides & $.0000(-.0002 ; .0002)$ & $.0000(-.0002 ; .0002)$ & $.0001(-.0003 ; .0002)$ & $-.0003(-.0001 ; .0002)$ \\
\hline Cardiometabolic risk score $^{1}$ & $.0003(-.0000 ; .001)$ & $.0003(.0000 ; .001)^{*}$ & $.0004(.0000 ; .001)^{*}$ & $.0002(-.0002 ; .001)$ \\
\hline \multicolumn{5}{|l|}{ Zero tolerance } \\
\hline WC & $.002(.000 ; .003)^{*}$ & $.002(.000 ; .003)^{*}$ & $.002(-.000 ; .004)$ & $.003(-.001 ; .006)$ \\
\hline BMI & $.001(-.000 ; .001)$ & $.001(.000 ; .001)^{*}$ & $.001(-.000 ; .002)$ & $.002(.000 ; .003)^{*}$ \\
\hline Glucose & $.0001(-.0001 ; .0004)$ & $.0001(-.0001 ; .0004)$ & $.0002(-.0002 ; .0001)$ & $.0002(-.0004 ; .001)$ \\
\hline C-peptide & $.0002(.0000 ; .0003)^{*}$ & $.0002(-.0000 ; .0003)$ & $.0001(-.0002 ; .0004)$ & $.0000(-.0005 ; .0004)$ \\
\hline Total cholesterol & $.0001(-.0002 ; .0005)$ & $.0002(-.0002 ; .0001)$ & $.0003(-.0003 ; .0001)$ & $.0003(-.001 ; .001)$ \\
\hline LDL-C & $.0002(-.0001 ; .0005)$ & $.0001(-.0001 ; .0001)$ & $.0004(-.0001 ; .0001)$ & $.0004(-.0003 ; .001)$ \\
\hline HDL-C & $.0001(-.0001 ; .0002)$ & $.0001(-.0001 ; .0002)$ & $.0000(-.0002 ; .0003)$ & $.0000(-.0004 ; .0004)$ \\
\hline Triglycerides & $.0000(-.0002 ; .0002)$ & $-.0001(-.0003 ; .0002)$ & $-.0030(-.0001 ; .001)$ & $-.0010(-.001 ;-.0002)^{*}$ \\
\hline Cardiometabolic risk score $^{1}$ & $.0003(.000 ; .001)^{*}$ & $.0003(.000 ; .001)^{*}$ & $.0004(-.0001 ; .001)$ & $.0003(-.0005 ; .001)$ \\
\hline
\end{tabular}

BMI, body mass index; LDL-C, low-density lipoprotein cholesterol; HDL-C, high-density lipoprotein cholesterol; WC, waist circumference.

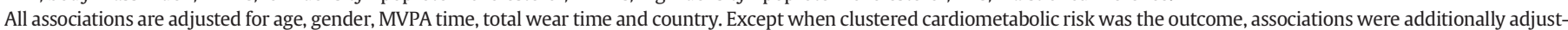
ed for waist circumference.

$1 \mathrm{~N}=111$.

* Indicates statistical significant associations $(\mathrm{p}<0.05)$.

\section{Discussion}

The present study examined the occurrence and duration of sedentary bouts when using various operational definitions of sedentary bouts in 10-13 year old children. Our findings reveal that variations in the operationalization of the definition of a sedentary bout have a large effect on estimates of sedentary time accumulated in bouts. Moreover, our findings suggest that children engage mostly in sedentary bouts of at least $5 \mathrm{~min}$, while bouts longer than $20 \mathrm{~min}$ are rare. We found few significant associations between total sedentary time/ sedentary time accumulated in bouts and cardiometabolic health indicators. Additionally, we found that applying the strictest definition of uninterrupted sedentary time accumulated in bouts (i.e. allowing zero tolerance time within bouts) resulted in more significant associations. Nevertheless, as we found a large number of non-significant associations, we conclude that we found no convincing evidence for an association between total sedentary time or sedentary time accumulated in bouts and cardiometabolic health indicators in 10-12 year old children.

Our finding that children predominantly spent their sedentary time in short bouts (i.e. bouts of at least 5 or $10 \mathrm{~min}$ ) is in line with previous studies (Saunders et al., 2013b; Carson et al., 2014; Harrington et al., 2011). In contrast to our finding that sedentary bouts longer than 20 min were rare among children, Colley et al. (2013) found that bouts of at least 20,40,60, 80, 100 and 120 min were common in 6-19 year old children on weekdays after 3 PM. This contrasting result could be explained by the difference in tolerance minutes that were allowed within sedentary bouts. Colley et al. (2013) tolerated up to $20 \%$ of minutes above $100 \mathrm{cpm}$, whereas we tolerated a maximum of $5 \%$ of minutes above $100 \mathrm{cpm}$ within bouts of at least $20 \mathrm{~min}$.
To date, few studies have been published on the association of sedentary bouts with cardiometabolic health in children. As stated in the introduction, these studies all used different operational definitions of sedentary bouts thereby making comparison of the findings complex (Carson and Janssen, 2011; Carson et al., 2014; Colley et al., 2013; Saunders et al., 2013b). Carson and Janssen (2011) found no association between sedentary time accumulated in bouts of at least $30 \mathrm{~min}$ and cardiometabolic risk factors in 6-19 year old children. Colley et al. (2013) found significant associations only between sedentary bouts of at least 40 and 80 min and anthropometrics in boys aged 10-14 years. No significant associations were found with other bout durations (i.e. 20, 60, 100 and $120 \mathrm{~min}$ ), or in younger (6-10 years) and older (15-19 years) boys, or in girls of any age, or with systolic and diastolic blood pressure and non-HDL cholesterol. Both studies allowed up to $20 \%$ of time $>100 \mathrm{cpm}$ within sedentary bouts (Colley et al., 2013; Carson and Janssen, 2011), thereby tolerating a substantial amount of time of non-sedentary time within sedentary bouts. This toleration of substantial interruptions in sedentary time may be an explanation for the lack of a consistent association with health indicators in these studies. Our finding that several associations became significant when applying the strictest definition of uninterrupted sedentary time accumulated in bouts (i.e. allowing zero tolerance time within bouts) supports this explanation. Moreover, this is in line with experimental evidence on the beneficial effects of brief interruptions during prolonged sitting in young adults (Altenburg et al., 2013; Peddie et al., 2013).

Carson et al. (2014) and Saunders et al. (2013b) examined delimited sedentary bout durations lasting 1-4 min, 5-9 min, 10-14 min, 15$29 \mathrm{~min}$ and $30+$ minutes (i.e. allowing zero tolerance time). After 
stratification for moderate-to-vigorous physical activity (MVPA), Carson et al. (2014) found significant associations of 5-9 and 1019 min bouts with body mass index standard deviation scores (BMI-Z scores) only in the low MVPA group, but not in the high MVPA group. No significant associations were found with other bout durations (i.e. 1-4, 20-29 and 30+ minutes) (Carson et al., 2014). Saunders et al. (2013b) found a significant negative association between sedentary bouts lasting 5-9 min and waist circumference in girls and significant positive associations between sedentary bouts of 10-14 min and BMI $\mathrm{z}$-score in boys and fasting glucose in girls. Sedentary bouts of 1519 min were negatively associated with fasting triglycerides and high sensitivity C-reactive protein. Using delimited bout durations make the findings of these studies hard to interpret and compare with our findings.

In the present study, we found few significant associations of sedentary time and cardiometabolic health indicators, but the number of significant associations increased when we applied the strictest definition of uninterrupted sedentary time. The lack of consistent associations between sedentary time accumulated in uninterrupted sedentary bouts and cardiometabolic health indicators may have two explanations. First, sedentary bouts longer than 20 min were rare in this group of 10-13 year old children, and as a consequence associations of such bouts with cardiometabolic health indicators may be underpowered. Second, children's exposure to excessive time spent in uninterrupted sedentary bouts may not have been long enough to demonstrate negative associations with cardiometabolic health indicators. Third, there is no association between sedentary bouts and cardiometabolic health indicators in this age group. Future prospective high-quality studies should examine the relationship between accumulated time spent in sedentary bouts during childhood and cardiometabolic health indicators during adulthood.

Strengths of this study include objectively assessed sedentary time and cardiometabolic health indicators, use of evidence-based accelerometer data reduction criteria, detailed analyses of sedentary time accumulated in bouts of uninterrupted (i.e. allowing zero tolerance time) and interrupted (i.e. allowing 30 and 60 s tolerance within sedentary bout) sedentary time and the relatively large sample of children for accelerometry and anthropometrics. One limitation of this study is the cross-sectional design, thereby limiting conclusions about causality. In addition, the sample size for the associations with fasting blood samples was small $(\mathrm{n}=112)$. Furthermore, as with all waist-worn accelerometer-based sedentary time, we were unable to differentiate between various postures (i.e. lying, sitting, standing). As a consequence of potential misclassification, time spent sedentary (both total and accumulated in bouts) may be overestimated.

\section{Conclusion}

This study demonstrates that different operational definitions of sedentary bouts lead to large differences in the occurrence and duration of children's sedentary time accumulated in bouts, and to differences in the cross-sectional association with children's cardiometabolic health indicators. We found that children accumulated their sedentary time predominantly in short bouts and this sedentary pattern was not convincingly associated with cardiometabolic health indicators in 10-13 year old children. As associations were more often significant when applying the strictest definition for sedentary bouts, and health effects may add up after extended periods of uninterrupted sedentary time (Altenburg et al., 2013; Peddie et al., 2013), we recommend that future prospective studies define a sedentary bout as a period of uninterrupted sedentary time, i.e. allowing no tolerance time within sedentary bouts.

Competing interest statement

None of the authors had a personal or financial conflict of interest.

\section{Funding}

The ENERGY-project is funded by the Seventh Framework Programme (CORDIS FP7, http://cordis.europa.eu/fp7/home_en.html) of the European Commission, HEALTH (FP7-HEALTH-2007-8). The contributions of TA and MC were funded by the Netherlands Organization for Health Research and Development (Grant number 91211057). MV is supported by the Research Foundation Flanders (FWO) (postdoctoral research fellowship: FWO13/PDO/191). The Swiss part of the ENERGYproject was funded by the Swiss Federal Office for Sport (BASPO) and the Swiss Federal Office of Public Health (BAG).

\section{Acknowledgments}

The ENERGY-project is funded by the Seventh Framework Programme (CORDIS) of the European Commission, HEALTH (FP7HEALTH-2007-B), Grant agreement no. 223254. The contributions of TA and MC were funded by the Netherlands Organization for Health Research and Development (Grant number 91211057). The Swiss part of the ENERGY-project was funded by the Swiss Federal Office for Sport (BASPO) and the Swiss Federal Office of Public Health (BAG). The results of the present study do not constitute endorsement by ACSM.

\section{References}

Altenburg, T.M., Hofsteenge, G.H., Weijs, P.J., Delemarre-Van de Waal, H.A., Chinapaw, M.J., 2012. Self-reported screen time and cardiometabolic risk in obese Dutch adolescents. PLoS One 7, e53333.

Altenburg, T.M., Rotteveel, J., Dunstan, D.W., Salmon, J., Chinapaw, M.J., 2013. The effect of interrupting prolonged sitting time with short hourly moderate-intensity cycling bouts on cardiometabolic risk factors in healthy young adults. J. Appl. Physiol. 115 (1985), 1751-1756.

Andersen, L.B., Harro, M., Sardinha, L.B., et al., 2006. Physical activity and clustered cardiovascular risk in children: a cross-sectional study (The European Youth Heart Study). Lancet 368, 299-304.

Bailey, D.P., Boddy, L.M., Savory, L.A., Denton, S.J., Kerr, C.J., 2013. Choice of activityintensity classification thresholds impacts upon accelerometer-assessed physical activity-health relationships in children. PLoS One 8, e57101.

Bey, L., Hamilton, M.T., 2003. Suppression of skeletal muscle lipoprotein lipase activity during physical inactivity: a molecular reason to maintain daily low-intensity activity. J. Physiol. 551, 673-682.

Brug, J., te Velde, S.J., Chinapaw, M.J., et al., 2010. Evidence-based development of schoolbased and family-involved prevention of overweight across Europe: the ENERGYproject's design and conceptual framework. BMC Public Health 10, 276

Carson, V., Janssen, I., 2011. Volume, patterns, and types of sedentary behavior and cardiometabolic health in children and adolescents: a cross-sectional study. BMC Public Health 11, 274.

Carson, V., Stone, M., Faulkner, G., 2014. Patterns of sedentary behavior and weight status among children. Pediatr. Exerc. Sci. 26, 95-102.

Chinapaw, M.J., Yildirim, M., Altenburg, T.M., et al., 2012. Objective and self-rated sedentary time and indicators of metabolic health in Dutch and Hungarian 10-12 year olds: the ENERGY-project. PLoS One 7, e36657.

Chinapaw, M.J.M., de Niet, M., Verloigne, M., de Bourdeaudhuij, I., Brug, J., Altenburg, T.M., 2014. From Sitting Time to Sitting Pattern: Accelerometer Data Reduction Decisions in Youth. PLoS One 9, e111205

Cole, T.J., Bellizzi, M.C., Flegal, K.M., Dietz, W.H., 2000. Establishing a standard definition for child overweight and obesity worldwide: international survey. BMJ 320, $1240-1243$.

Colley, R.C., Garriguet, D., Janssen, I., et al., 2013. The association between accelerometermeasured patterns of sedentary time and health risk in children and youth: results from the Canadian Health Measures Survey. BMC Public Health 13, 200.

Dunstan, D.W., Kingwell, B.A., Larsen, R., et al., 2012. Breaking up prolonged sitting reduces postprandial glucose and insulin responses. Diabetes Care 35, 976-983.

Fischer, C., Yildirim, M., Salmon, J., Chinapaw, M.J., 2012. Comparing different accelerometer cut-points for sedentary time in children. Pediatr. Exerc. Sci. 24, 220-228.

Gootjes, J., Tel, R.M., Bergkamp, F.J., Gorgels, J.P., 2009. Laboratory evaluation of a novel capillary blood sampling device for measuring eight clinical chemistry parameters and HbA1c. Clin. Chim. Acta 401, 152-157.

Hamilton, M.T., Hamilton, D.G., Zderic, T.W., 2004. Exercise physiology versus inactivity physiology: an essential concept for understanding lipoprotein lipase regulation. Exerc. Sport Sci. Rev. 32, 161-166.

Harrington, D.M., Dowd, K.P., Bourke, A.K., Donnelly, A.E., 2011. Cross-sectional analysis of levels and patterns of objectively measured sedentary time in adolescent females. Int. J. Behav. Nutr. Phys. Act. 8, 120.

Peddie, M.C., Bone, J.L., Rehrer, N.J., Skeaff, C.M., Gray, A.R., Perry, T.L., 2013. Breaking prolonged sitting reduces postprandial glycemia in healthy, normal-weight adults: a randomized crossover trial. Am. J. Clin. Nutr. 98, 358-366.

Robusto, K.M., Trost, S.G., 2012. Comparison of three generations of ActiGraph activity monitors in children and adolescents. J. Sports Sci. 30, 1429-1435. 
Saunders, T.J., Chaput, J.P., Goldfield, G.S., et al., 2013a. Prolonged sitting and markers of cardiometabolic disease risk in children and youth: a randomized crossover study. Metabolism 62, 1423-1428.

Saunders, T.J., Tremblay, M.S., Mathieu, M.E., et al., 2013b. Associations of sedentary behavior, sedentary bouts and breaks in sedentary time with cardiometabolic risk in children with a family history of obesity. PLoS One 8, e79143.

Treuth, M.S., Schmitz, K., Catellier, D.J., et al., 2004. Defining accelerometer thresholds for activity intensities in adolescent girls. Med. Sci. Sports Exerc. 36, 1259-1266.
Trost, S.G., Loprinzi, P.D., Moore, R., Pfeiffer, K.A., 2011. Comparison of accelerometer cut points for predicting activity intensity in youth. Med. Sci. Sports Exerc. 43, 1360-1368. van Stralen, M.M., te Velde, S.J., Singh, A.S., et al., 2011. EuropeaN Energy balance Research to prevent excessive weight Gain among Youth (ENERGY) project: design and methodology of the ENERGY cross-sectional survey. BMC Public Health 11, 65.

Yildirim, M., Verloigne, M., de, B.I., et al., 2011. Study protocol of physical activity and sedentary behaviour measurement among schoolchildren by accelerometry-crosssectional survey as part of the ENERGY-project. BMC Public Health 11, 182. 